\title{
A Machine Learning Approach to the Prediction of Fretting Fatigue Life
}

\author{
Nowell, D. ${ }^{*}$ and Nowell, P.W. ${ }^{2}$
}

Abstract

The paper analyses some fretting fatigue results from the literature, reported by Nowell and by Szolwinski and Farris. The principal variables of contact size, peak pressure, remote specimen tension, and tangential force ratio are identified and these are used to construct an Artificial Neural Network (ANN), aimed at predicting total fretting fatigue life. The network is trained and validated using $90 \%$ of the data, and its success at predicting the results for the remaining $10 \%$ of unseen data is examined. The network is found to be very effective at separating the results into low life and 'run-out' groups. It is less successful at predicting lives for the low life specimens, but this is largely due to the difficulty of incorporating the runout and finite life tests together in the same dataset. The approach is seen to be potentially useful and identifies contact size as a key variable. However, the results highlight the need for significant numbers of experimental results if the method is to be used effectively in future. Nevertheless, the trained network comprises a useful tool for the prediction of future experimental results with this material.

\section{Introduction}

Fretting fatigue occurs when two contacting surfaces carry a varying tangential load, such as is the case in many engineering systems. The micro-slip, which is almost unavoidable, leads to characteristic fretting marks on the contacting surfaces [1], and frequently cracks will initiate close to the edge of the contact, due to the severe stress concentration [2]. There have been many attempts to accurately predict fretting fatigue life over a range of conditions. However, there is no universally accepted method, appropriate to industrial situations, which occur in a wide range of applications. Indeed, Dobromirski [3] highlights the complexity of the situation by discussing whether up to 50 variables are required in order to fully characterise a fretting fatigue problem. Hence, in building models which are useful in practical situations, the researcher frequently has to start with the question of which aspects to include and which to leave out.

Machine learning techniques are increasingly being used to analyse large engineering data sets and potentially they can help to identify the key variables in a problem. Different approaches are used, including, regression, clustering, and artificial neural networks. A number of authors (e.g. Durodola et al. [4] and Rovinelli et al. [5]) have used the approach to tackle fatigue problems. Recently, Pestana et al. [6] have used an artificial neural network (ANN) to predict the effect of mean stress on the fatigue of overhead conductors. Since this is a situation which may involve interwire fretting, it therefore seems appropriate to investigate the extent to which machine learning can provide insight into fretting fatigue phenomena. We also intend to examine the extent to which it can be used as a practical tool for predicting component life. As an initial investigation, we will revisit the results provided by Nowell [7], who reported fretting fatigue lives under carefully-controlled conditions, and which have been used as a standard database by a number of authors (e.g. Ciavarella [8]) in comparing different life prediction methods. It should be noted that a particular focus of Nowell's experiments was to investigate the effect of contact size (and therefore of stress gradient) on the fretting fatigue performance of an Al4\%Cu alloy.

\footnotetext{
${ }^{1}$ Imperial College London, Exhibition Rd, London SW7 2AZ

2 University of Reading, Reading, Berkshire, RG6 6AH

*Corresponding author, d.nowell@imperial.ac.uk
} 
The concept of artificial neural networks is probably familiar to many readers. They are based on an analogue with the human brain: inputs are connected to outputs via 'neurons' which are activated in response to the sum of their input. Weights are then adjusted to give a better fit to a set of 'training' data. Once this has been achieved, the trained network may be used to predict different combinations of inputs from those in the training data. Widrow, Rumelhart and Lehr [9] argue that most ANN applications fall into the following three categories:

Pattern classification (e.g. image analysis)

Prediction and financial analysis (e.g. weather forecasting)

Control and Optimization (e.g. the travelling salesman problem)

The prediction of fretting fatigue life falls into the second of these broad categories, although as we will see later on, there are elements of the first, which arise from the actual data set analysed. It should be noted that an alternative way of viewing the technique is that it is performing a 'best fit' surface fit to the experimental data as a function of the chosen variables. There are, of course, other means of achieving this, and it might be possible to fit a polynomial surface, or some other interpolating function to the data. However, ANN method does offer some advantages. Firstly, standard techniques and tools are available, which one can draw on. Secondly, one does not need to assume a particular form of the interpolating function in advance, the network itself will choose an appropriate solution, based on the training data. Hence, to some extent one can regard the approach as a more automated means of analysing the data.

\section{Experiments and modelling}

Nowell [7] carried out a series of experiments using cylindrical pads pressed against a flat specimen, as shown schematically in Fig. 1. The material used was an $\mathrm{Al} 4 \% \mathrm{Cu}$ alloy, commonly used in aerospace. The main aim of the study was to investigate a size effect, previously reported by Bramhall [10] where the life was found to be very sensitive to contact size. In order to do this, he exploited a feature of Hertzian contact, which allows the contact size and the peak pressure to be varied independently. The peak pressure, $p_{0}$, in a cylindrical Hertzian contact is given by [11] as

$$
p_{0}=\sqrt{\frac{P E^{*}}{\pi R}}
$$

Where $P$ is the normal load, $R$ the radius of the pads, and $E^{*}$ is the usual combination of elastic constants, which for similar bodies in contact is given by

$$
E^{*}=\frac{E}{2\left(1-v^{2}\right)}
$$

Where $E$ is Young's Modulus and $v$ is Poisson's ratio. Similarly, the contact semi-width, $a$, is given by

$$
a=\sqrt{\frac{4 P R}{\pi E^{*}}}
$$

Hence, it may readily be seen that varying $P$ and $R$ so that the ratio $P / R$ is constant varies the size of the contact and hence the spatial extent of the stress field whilst maintaining the magnitude of the stresses (governed by $p_{o}$ ) constant. A typical set of results from [7] is plotted in Fig. 2 . This shows the variation of total fretting fatigue life with contact size. The symbols with arrows are 'run-outs' where the specimen remained unbroken after $10^{7}$ fatigue cycles. It may be seen that small contacts led to run-outs, whereas larger contacts failed after about $10^{6} \mathrm{cycles}$. 


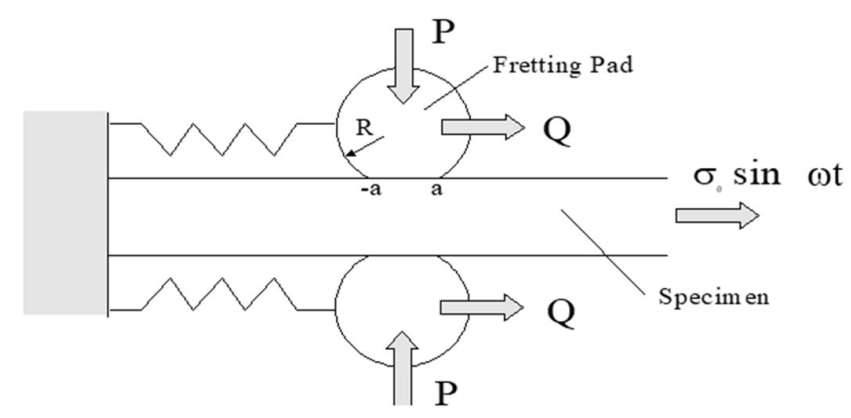

Figure 1. - Schematic of Nowell's experiments [7].

Nowell's experiments give 36 usable experimental results for our current purposes, of which 19 were run-outs at $10^{7}$ cycles. This is not sufficient to train, validate, and check a network, but additional data are also available from Szlowinski and Farris [12], who used the same geometrical configuration and a very similar material. This gave a further 37 experimental results, most of which are at lower fatigue lives than those in [7]. Figure 3. shows the results of all 73 experiments, plotted against the peak elastic stress, which occurs at the trailing edge of the contact. It can be seen that there is very little correlation between the peak stress and the life, meaning that a purely stressbased parameter is unlikely to be satisfactory for fatigue life prediction. The principal reason for the lack of correlation with a single variable will be shown to be the size effect investigated in [7]. Full details of the experiments may be found in [7] and [12], but the key parameters and experimental lives are reproduced here in Appendix 1 for convenience.

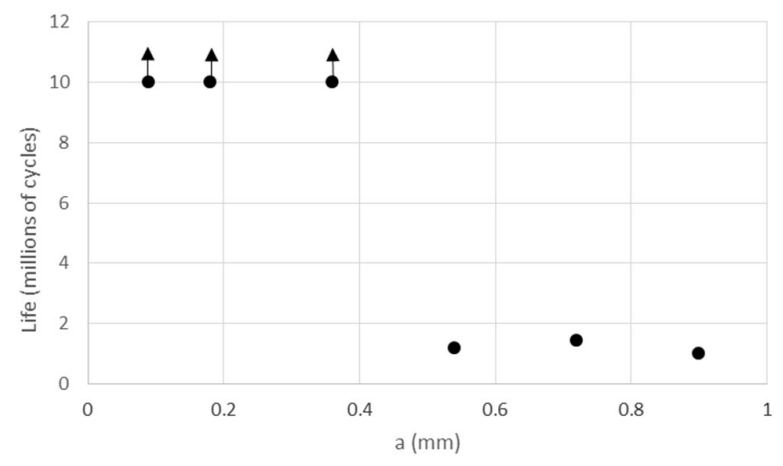

Figure 2. - Typical set of data from [7], showing the variation of fatigue life with contact size.

The simplest neural network that we can envisage takes the key inputs, which vary between the experiments $p_{0}, a$, the peak remote stress in the specimen, $\sigma_{0}$, and the tangential force ratio, $Q / P$ and relates them to a single output variable, fatigue life. In general we might also expect the life to vary with coefficient of friction, but we cannot include this here, since the value is the same for all experiments $(\sim 0.65)$. The reason for choosing these parameters is that they are quantities which may easily be measured in the experiment, or derived using simple formulae. Hence, the user should readily be able to predict the life under a different set of experimental conditions from the trained network, without further calculation. A single hidden layer of neurons is used between the inputs and the output. The overall architecture of such a network is shown in Figure 4. During the training phase the weights of the links (equivalent to synapses in the brain) are optimised to minimise the error between output and experimental life. It is relatively straightforward to write the required code from scratch [14], but there is also a wide range of public domain software available. 
For the current project we used 'JustNN' [15], principally because of the ease of control and for the range of graphical output available.

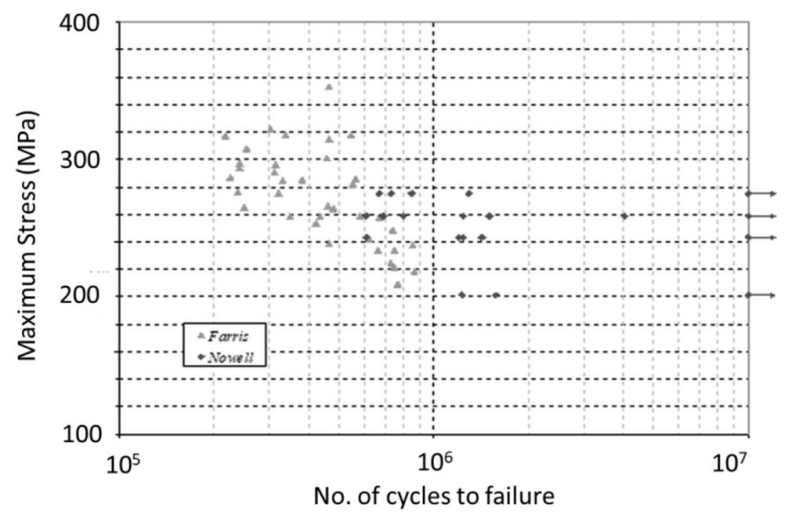

Figure 3. - Results from the experiments of Nowell [7] and Szlowinski and Farris [12] plotted against peak elastic stress (from [13]).

In order to apply the neural network approach, the data need to be divided into three groups: (i) Training data; (ii) Validation data; and (iii) Query examples. Seventy percent of the experimental results are randomly chosen for training. During this phase the error between the outputs of the network and the real experimental lives is minimised by adjusting the weights of the synapses in the network. A further $20 \%$ of the results are used for validation. This phase is important, as it explores how good the solution is at predicting the result on data that has not been used for training. If the result with the validation data is poor, the parameters used for training the network are adjusted so as to improve the results for the validation data set. If these have not been set correctly it is possible to 'over-fit' the training data, resulting in poor predictions on unseen data. This may be likened to using too high an order of polynomial to fit $(x, y)$ data in a conventional least squares minimisation. Query examples are kept until the overall network has been optimised, and are used to assess how good the network is at predicting the results for entirely unseen data. It is important that the network is not changed further as a result of examining performance on the Query data, as this may bias the network for future unseen data. The overall purpose of the network is to use the entire dataset to produce the best predictor for any further set of conditions. In order to do this it is usual to split the data into the $70 / 20 / 10$ proportions that we have used here. Other proportions can, of course, be used but since the purpose of the paper is to explore the use of the technique on fretting data, we have not chosen to investigate this here. In any case, reducing the training data is likely to give a worse predictive network, as it is not as comprehensively trained. 


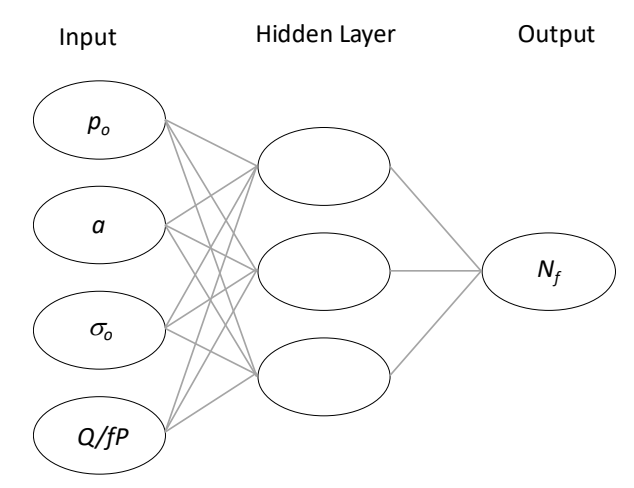

Figure 4. - Architecture of a simple neural network for the current problem, with a single layer of hidden nodes.

During the training phase the weights of the links (equivalent to synapses) are optimised to minimise the error between output and experimental life. These weights are stored as a synaptic matrix, and may be examined at the end of the optimisation. Optimisation is controlled by two parameters: the learn rate and the momentum [14]. The learn rate, $\eta$, controls how fast the network learns in each iteration or 'epoch'. The weights, $W$, are updated so that

$$
W_{n+1}=W_{n}-\eta \nabla \mathcal{L}
$$

where $\nabla \mathcal{L}$ is the derivative of the cost function representing the error between the output of the network and the experimental results. Thus, a low learn rate leads to larger number of epochs before convergence, but generally gives better stability. Increasing the learn rate reduces the number of epochs required, but is more likely to lead to convergence problems. The momentum parameter, $h$, is used to avoid local minima. Once again, an optimum choice exists between a low value, which may allow the output to become 'stuck' in a local minimum, and a high value, which may miss the global minimum, giving a poor solution. The default values of 0.6 for the learn rate and 0.8 for the momentum in the JustNN software were used for the initial training of the network.

\section{Results and Discussion}

An initial run was conducted for 400 epochs with the default values of $\eta$ and $h$ given above and the results are displayed in Fig. 5. Fifty-two training examples were used, together with 15 validation cases. Figure 5 plots the maximum, mean, and minimum normalised training error against epoch number. It may be seen that the mean training error decreases rapidly during the first 70 epochs, together with the maximum error. This improvement is achieved by adjusting the weights in the network. After 100 cycles the validation data is introduced. This shows an initial error of approximately 0.05 on the validation data, but it does not improve much as further adjustment of the network is carried out. Hence, after 400 epochs, only 8 of the 15 validation predictions are within $10 \%$ of the experimental value. This confirms that most of the training of the network had taken place before the validation data is examined. Further, it suggests that the process may have over-fitted the training data, failing to identify overall trends and as a result performing badly on the validation data. 


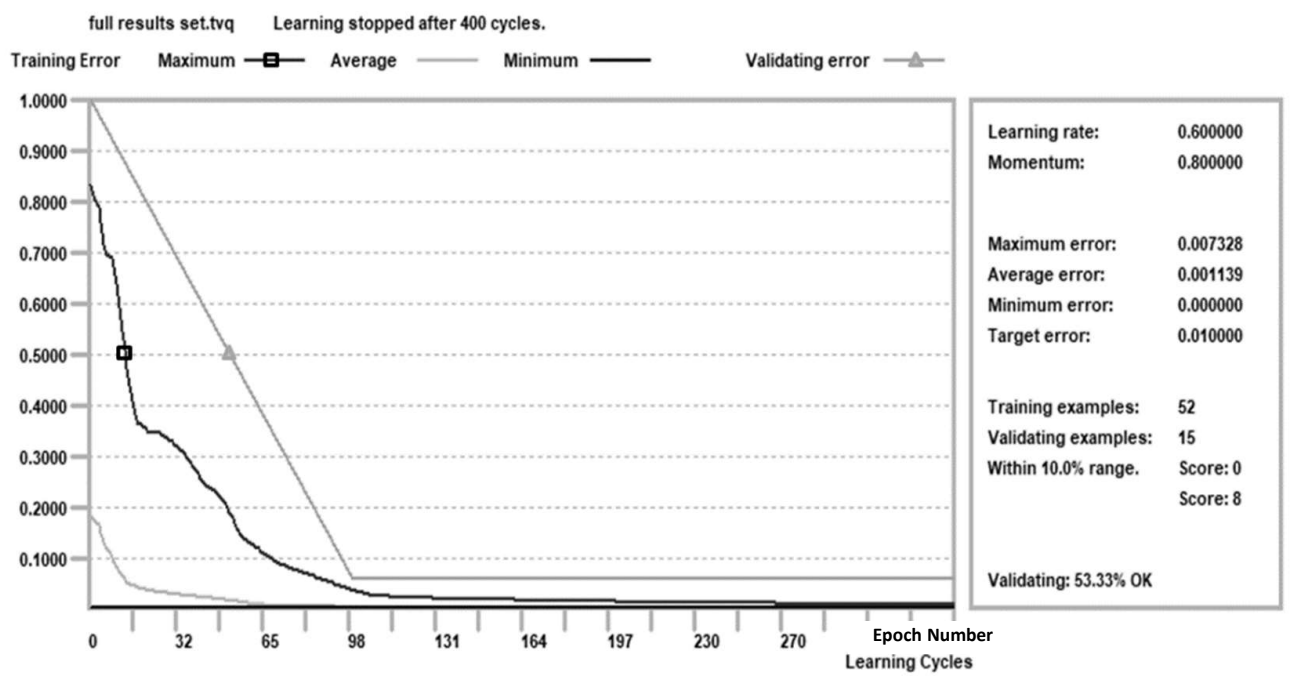

Figure 5. - Initial output from the network $(\eta=0.6$ and $h=0.8)$. Normalised training error vs epoch number.

An improved result may be obtained by adjusting some of the network parameters. The learning rate is reduced to 0.2 , and the momentum to 0.5 . Further, the network is allowed to run for longer, up to 4000 epochs. Figure 6. shows the equivalent result to Fig. 5. As expected, learning is much slower, but the number of validation examples within $10 \%$ of the experimental value has increased to $12(80 \%)$. It is now possible to examine the effect of $\eta$ and $h$ more systematically. Varying $\eta$ between 0.1 and 0.9 produces 12 validation examples within $10 \%$ in each case, suggesting that the change in $h$ which is largely responsible for the improvement between Figures 5 and 6 . A series of runs were carried out with $\eta=0.5$ and varying $h$ between 0.1 and 0.9 . Values of $h$ between 0.1 and 0.7 again produced 12 accurate validation predictions, but this falls to 10 when $h=0.8$ and 4 when $h$ $=0.9$. Hence, it seems that the results are fairly insensitive to $\eta$ and $h$, provided that $h$ is kept below 0.7. Hence, our final choice of values were $\eta=0.5, h=0.5$. Output for these values is shown in Fig. 7.

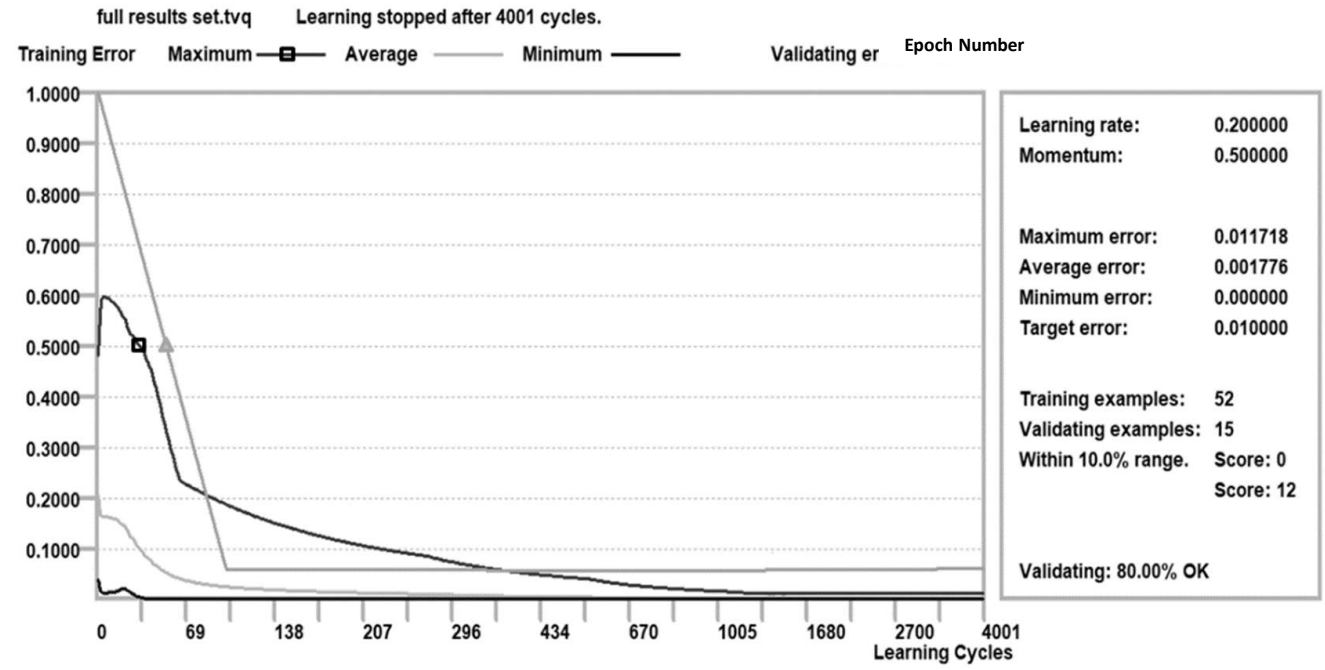

Figure 6. - Revised output from the network. Normalised training error vs epoch number for $\eta=0.2$ and $\mathrm{h}=0.5$. Note the compressed horizontal scale. 


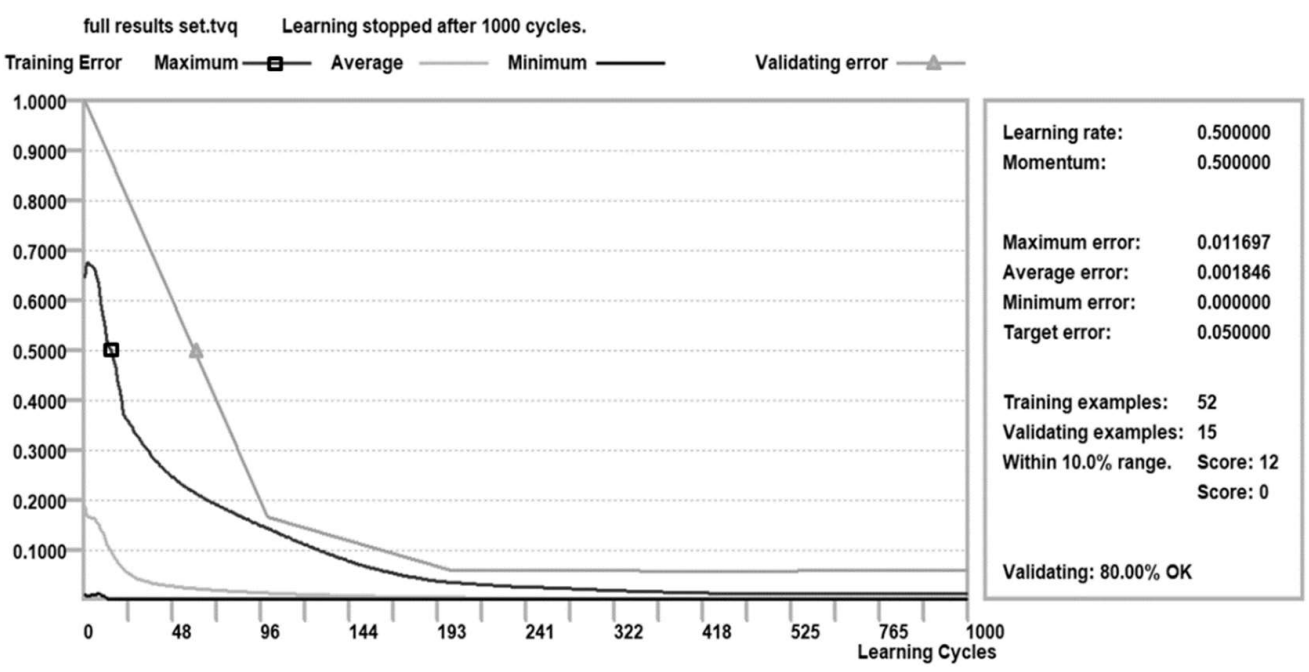

Figure 7. - Final output from the network. Normalised training error vs epoch number for $\eta=0.5$ and $\mathrm{h}=0.5$.

The optimum choice of these parameters will depend on the nature of the dataset, which is precisely why they are left adjustable in the implementation. Conceptually it would be possible to interface the ANN software to an optimiser so that these parameters could be adjusted according to some agreed objective function. However, as noted above the solution is not particularly sensitive to the choice, as long as reasonable values are chosen. What can vary is the number of iterations taken to reach the solution. Detailed discussion of these points is beyond the scope of the current paper and the reader is therefore referred to standard text books on machine learning, such as [14] for further clarification.

It is now appropriate to examine the results of the final network in more detail. First, Fig. 8. shows a schematic of the final network. It will be seen that this has only 2 hidden neurons, as opposed to 3 shown in Fig. 4. The JustNN software automatically 'grows' the number of hidden neurons in order to produce a good solution without over-fitting. The width of the synapsis links in the figure show the relative weights of the connections in the network. The relative importance of the input variables may then be calculated. This gives the most significant parameter as the contact semiwidth, $a(1.00)$, followed by the remote stress $\sigma_{0}(0.31)$ and $Q / P(0.28)$. Finally, the effect of $p_{0}$ is found to be relatively insignificant (0.02). Had we chosen a different set of input parameters, then it is possible that the contact size would not appear so dominant, but we need to bear in mind that Nowell's original experiments were designed specifically to investigate contact size effects, hence it is perhaps not entirely surprising that this parameter emerges with the highest weight. 


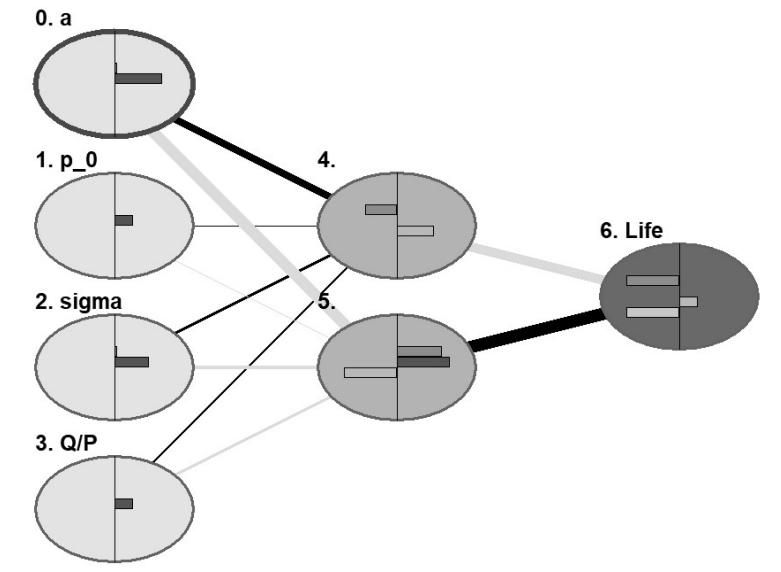

Figure 8. - Schematic of the final network with line thicknesses indicating the importance of the links. Grey links signify positive weights and black links negative weights.

Once the final network has been configured it can be applied to the remaining query dataset. This consists of six experimental results which are unseen during the training and validation of the network. A comparison between the experimental lives and those predicted by the network is given in Fig. 9. It will be seen that it consists of five experiments which lie in the short life regime, one which is a run-out at $10^{7}$ cycles, and an intermediate result with an experimental life of $5 \times 10^{6}$ cycles. The network has been successful at predicting the run-out result, and is moderately successful at predicting the low-life results. However the intermediate result has a predicted life which is only $45 \%$ of the experimental value. The results highlight one issue with the current data set, namely that it largely comprises low life results and run-outs. There are very few results which lie in between these two extremes. The network is very good at categorising results into low and high life results, but is less good at those in-between, because of the lack of training data in this region.

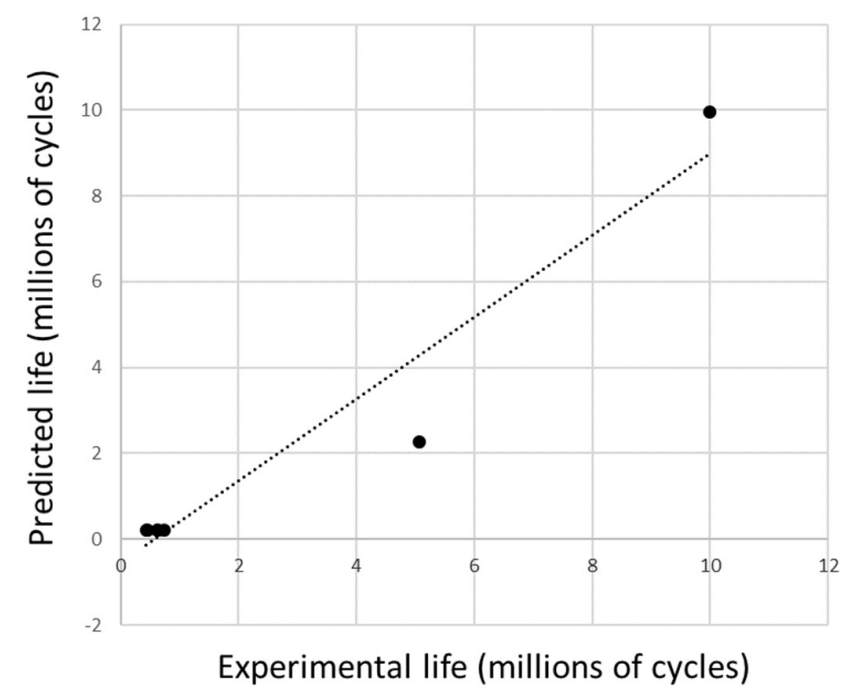

Figure 8. - Comparison between predicted and experimental lives for the query data set using the parameters shown in Fig. 7. 
Once the network has been trained and we have examined the validation and query data, we can now use it to predict further results. Figure 9 shows a comparison between the experimental results for Nowell's Series 4 experiments, and the output from the ANN across the whole range of a. Once again, there is a good prediction of the transition contact size, where the lives suddenly become finite, but predictions in the low life regime are less good. This highlights a weakness in the approach in that the original data contain run outs, where we only know that the life is greater than $10^{7}$ cycles, as the experiment is then stopped. In formulating the input to the ANN we cannot readily distinguish between run outs and finite lives. Hence, a figure of $10^{7}$ is input for the life of the runouts, and the ANN attempts to fit to this, assuming that it is a finite life (see Fig. 9.).A more robust approach might be to disregard the runout results and to focus on the remaining 54 tests. However, this now leaves us with relatively few experimental results to train and validate the network. Further, it would miss the point of Nowell's original experiment, i.e. to investigate the size effect, by eliminating the small contacts which give rise to long lives. Hence it does not seem worthwhile to explore this further.

The trained network is able to predict the life for any set of experimental conditions. For example, we can examine the effect of varying peak pressure, with all of the other parameters held constant. Example results are shown in Fig. 10, where the peak pressure (and therefore the overall normal load) is varied with the other parameters kept constant ( $a=0.9 \mathrm{~mm}, \sigma_{0}=93 \mathrm{MPa}$, and $\left.\mathrm{Q} / \mathrm{P}=0.45\right)$. It can be seen that the network predicts a modest increase in life with increasing normal load, as the increased compression due to the contact provides some protection against the tensile stress in the specimen.

\section{Conclusions}

An artificial neural network has been built, taking the inputs of contact size, peak pressure, specimen tensile stress and tangential force ratio. The network has been trained on fretting fatigue data produced by Nowell [7] and by Szolwinski and Farris [12] for a range of these parameters. It has been shown that it can predict the outcome of the experiments in terms of total life with reasonable accuracy. The importance of the contact size has once again been highlighted, and this has been shown to be the most significant input parameter. Peak pressure was found to be the least significant, with the other two lying in between. Once practical consequence of this is that attempts to carry out life prediction for fretting fatigue based on peak stress alone (i.e. without including a length scale) would seem destined to failure. The results allow prioritisation of the important parameters for more physically-based models and highlight the importance of including a length scale in the input data. The data set examined is perhaps not typical, as it contains a mix of long and short life data, with not many results in between. However the network is very successful at classifying into these two groups. In this respect the problem turns out to be more of a type 1 problem according to Widrow, Rumelhart and Lehr [9], rather than the expected type 2 (prediction) problem. It would be interesting to extend the approach to other materials and to other geometries, but reliable data in the literature is very sparse. Fretting fatigue tests are complex and time consuming to carry out, and approximately 100 results are appropriate to train and validate the network for 4 or 5 input parameters. It is extremely difficult to find such a database in the literature, and this presents a future challenge to the fretting community: can we develop reasonably large, well-characterised databases of results for a range of relevant materials in standard geometries, and how might such an enterprise be resourced?

Overall the machine learning approach presents a novel and useful alternative to traditional methods of examining fretting fatigue data. It has the advantage that one does not have to assume anything about the relative importance of the different variables, a priori. The results of the investigation will highlight this, and will therefore produce a foundation for more detailed analysis. 
On the other hand, a criticism of the technique might be that a relatively large number of test results are required in order to train the network. Further, a criticism might be that the method is essentially 'black box' in nature. It is difficult to incorporate any prior understanding of the physics of the problem, beyond the choice of the input variables.

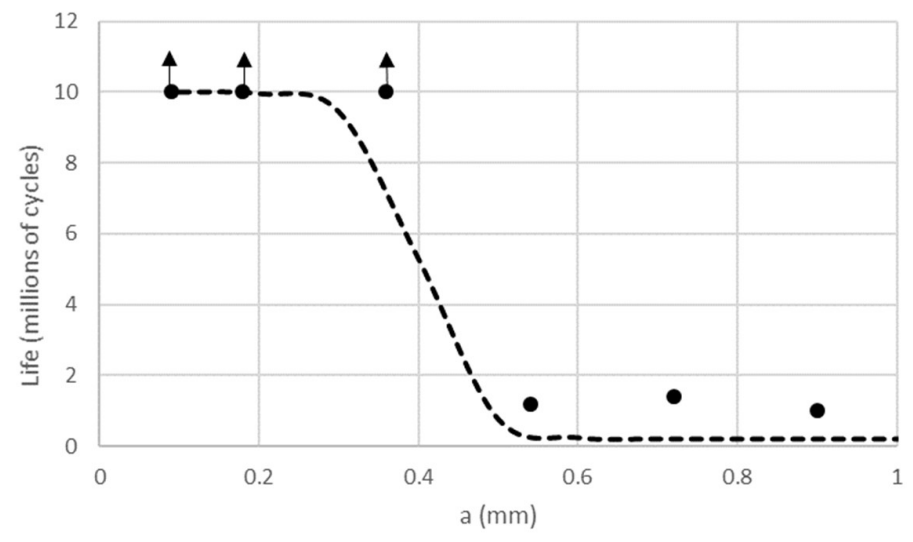

Fig. 9. - Comparison between Nowell's results for Series 4 ( $p_{0}=143 \mathrm{MPa}, \sigma_{0}=77.2 \mathrm{MPa}, \mathrm{Q} / \mathrm{P}=0.45$ ) (points) against ANN predictions (dotted line)

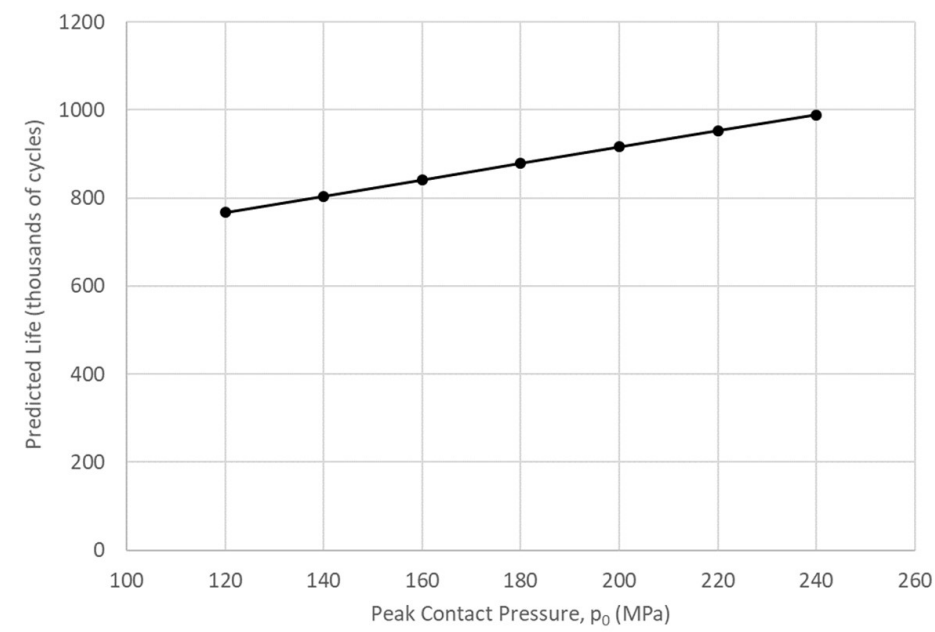

Fig. 10. Predicted lives as a function of peak contact pressure $p_{0}\left(a=0.9 \mathrm{~mm}, \sigma_{0}=93 \mathrm{MPa}\right.$, and

$$
Q / P=0.45)
$$

\section{References}

1. Waterhouse, R.B., 'Fretting Corrosion', Pergamon Press, Oxford, 1972.

2. Hills, D.A., and Nowell, D. 'Mechanics of Fretting Fatigue', Kluwer, Dordrecht, 1994.

3. Dobromirski, J.M., 'Variables of Fretting Process, Are there 50 of them?' pp 60-66 of 'Standardization of Fretting Fatigue Test Methods and Equipment, ASTM STP 1159', M.H. Attia and R.B. Waterhouse, Eds, ASTM, Philadelphia, 1992. 
4. Durodola, J.F., Li, N., Ramachandra, S. and Thite, A.N., 'A pattern recognition artificial neural network method for random fatigue loading life prediction', International Journal of Fatigue, 99, 55-67, 2017.

5. Rovinelli, A., Sangid, M.D. Proudhon, H. and Ludwig, W., 'Using machine learning and a datadriven approach to identify the small fatigue crack driving force in polycrystalline materials', Computational Materials, 4, Article number: 35, 2018.

6. Pestana, M.S., Kalombo, R.B., Freire, R.C.S. Jr, Ferreira, J.L.A., da Silva, C.R.M., and Araújo, J.A., 'Use of artificial neural network to assess the effect of mean stress on fatigue of overhead conductors', Fatigue Fract Eng Mater Struct. 41(12), 2577-2586, 2018.

7. Nowell, D., 'An Analysis of Fretting Fatigue', D.Phil. thesis, University of Oxford, 1988.

8. Ciavarella, M., and Dini, D., 'A refined CLNA model in fretting fatigue using asymptotic characterization of the contact stress fields', Fatigue and Fracture of Engineering Materials and Structures, 28, 1099-1112, 2005.

9. Widrow, B., Rumelhart, D.E. and Lehr, M.A., 'Neural networks: applications in industry, business and science'. Communications of the ACM, 37(3), 93-106, 1994.

10. Bramhall, R., 'Studies in Fretting Fatigue', D.Phil. thesis, University of Oxford, 1973.

11. Johnson, K.L., 'Contact Mechanics', Cambridge University Press, 1985.

12. Szolwinski, M.P., and Farris, T.N., 'Observation, analysis and prediction of fretting fatigue in 2024-T351 aluminum alloy', Wear 221(1), 24-36, 1998.

13. Dini, D., 'Studies in fretting fatigue with application to almost complete contacts', D.Phil. thesis, University of Oxford, 2004.

14. Rascha, S. and Mirjalili, V., 'Python Machine Learning', Second Edition, Packt Publishing, Birmingham, 2017.

15. Neural Planner Software, 'JustNN - Free Neural Network Software', www.justnn.com, 2015, accessed 4/6/19. 


\section{Appendix 1 - Experimental data}

Table 1 - Fretting fatigue results from Nowell [7]

\begin{tabular}{|l|l|l|l|l|l|}
\hline Expt & $\mathrm{a}(\mathrm{mm})$ & $\mathrm{p}_{0}(\mathrm{MPa})$ & $\sigma_{0}(\mathrm{MPa})$ & $\mathrm{Q} / \mathrm{P}$ & Life (Millions of Cycles) \\
\hline 1.1 & 0.38 & 157 & 92.7 & 0.45 & 1.29 \\
\hline 1.2 & 0.57 & 157 & 92.7 & 0.45 & 0.67 \\
\hline 1.3 & 0.95 & 157 & 92.7 & 0.45 & 0.73 \\
\hline 1.4 & 0.76 & 157 & 92.7 & 0.45 & 0.85 \\
\hline 1.5 & 1.14 & 157 & 92.7 & 0.45 & 0.67 \\
\hline 1.6 & 0.1 & 157 & 92.7 & 0.45 & $>10$ \\
\hline 1.7 & 0.19 & 157 & 92.7 & 0.45 & $>10$ \\
\hline 1.8 & 0.28 & 157 & 92.7 & 0.45 & $>10$ \\
\hline 2.1 & 1.08 & 143 & 92.7 & 0.24 & 1.28 \\
\hline 2.2 & 0.54 & 143 & 92.7 & 0.24 & $>10$ \\
\hline 2.3 & 0.18 & 143 & 92.7 & 0.24 & $>10$ \\
\hline 2.4 & 0.36 & 143 & 92.7 & 0.24 & $>10$ \\
\hline 2.5 & 0.09 & 143 & 92.7 & 0.24 & $>10$ \\
\hline 2.6 & 0.9 & 143 & 92.7 & 0.24 & 1.22 \\
\hline 2.7 & 0.72 & 143 & 92.7 & 0.24 & 5.06 \\
\hline 3.1 & 0.72 & 143 & 92.7 & 0.45 & 0.61 \\
\hline 3.2 & 0.9 & 143 & 92.7 & 0.45 & 1.24 \\
\hline 3.3 & 1.08 & 143 & 92.7 & 0.45 & 0.69 \\
\hline 3.4 & 0.36 & 143 & 92.7 & 0.45 & 1.5 \\
\hline 3.5 & 0.54 & 143 & 92.7 & 0.45 & 0.8 \\
\hline 3.6 & 0.18 & 143 & 92.7 & 0.45 & $>10$ \\
\hline 3.7 & 0.27 & 143 & 92.7 & 0.45 & 4.04 \\
\hline 3.8 & 0.09 & 143 & 92.7 & 0.45 & $>10$ \\
\hline 4.1 & 0.54 & 143 & 77.2 & 0.45 & 1.2 \\
\hline 4.2 & 0.36 & 143 & 77.2 & 0.45 & $>10$ \\
\hline 4.3 & 0.9 & 143 & 77.2 & 0.45 & 1.02 \\
\hline 4.4 & 0.72 & 143 & 77.2 & 0.45 & 1.42 \\
\hline 4.5 & 0.18 & 143 & 77.2 & 0.45 & $>10$ \\
\hline 4.6 & 0.09 & 143 & 77.2 & 0.45 & $>10$ \\
\hline 5.1 & 0.57 & 120 & 61.8 & 0.45 & $>10$ \\
\hline 5.2 & 0.71 & 120 & 61.8 & 0.45 & 1.57 \\
\hline 5.3 & 0.28 & 120 & 61.8 & 0.45 & $>10$ \\
\hline 5.4 & 0.14 & 120 & 61.8 & 0.45 & $>10$ \\
\hline 5.5 & 0.85 & 120 & 61.8 & 0.45 & 1.23 \\
\hline 5.6 & 0.21 & 120 & 61.8 & 0.45 & $>10$ \\
\hline 5.7 & 0.42 & 120 & 61.8 & 0.45 & $>10$ \\
\hline 5.8 & 0.21 & 120 & 61.8 & 0.45 & $>10$ \\
\hline & & & & & \\
\hline & & & & & \\
\hline
\end{tabular}


Table 2 - Fretting Fatigue results from Szolwinski and Farris [12]

\begin{tabular}{|l|l|l|l|l|l|}
\hline Expt & $\mathrm{a}(\mathrm{mm})$ & $\mathrm{p}_{0}(\mathrm{MPa})$ & $\sigma_{0}(\mathrm{MPa})$ & $\mathrm{Q} / \mathrm{P}$ & Life (Millions of Cycles) \\
\hline p001 & 1.54 & 246 & 110.3 & 0.22 & 0.31 \\
\hline p004 & 1.24 & 197.8 & 84.7 & 0.28 & 0.42 \\
\hline p006 & 1.31 & 208.4 & 110.3 & 0.31 & 0.24 \\
\hline p008 & 1.21 & 202.7 & 100.7 & 0.35 & 0.24 \\
\hline p011 & 1.37 & 230.6 & 110.3 & 0.31 & 0.22 \\
\hline MAF1a & 1.76 & 155.7 & 111.7 & 0.43 & 0.24 \\
\hline MAF1b & 1.75 & 155.3 & 112.9 & 0.37 & 0.25 \\
\hline MAF2 & 1.4 & 232 & 84.8 & 0.23 & 0.67 \\
\hline MAF3a & 1.66 & 189 & 100 & 0.27 & 0.34 \\
\hline MAF3b & 1.66 & 189 & 100 & 0.27 & 0.43 \\
\hline MAF4 & 1.3 & 207 & 88.4 & 0.35 & 0.56 \\
\hline MAF5a & 1.51 & 240 & 101.9 & 0.31 & 0.54 \\
\hline MAF5b & 1.51 & 240 & 101.9 & 0.31 & 0.33 \\
\hline MAF6 & 1.53 & 174 & 85.8 & 0.38 & 0.58 \\
\hline MAF7 & 1.88 & 166 & 97 & 0.32 & 0.74 \\
\hline MAF8 & 1.75 & 200 & 113.1 & 0.34 & 0.46 \\
\hline MAF9 & 1.88 & 167 & 85.4 & 0.32 & 0.86 \\
\hline MAF9 & 1.88 & 167 & 85.4 & 0.32 & 0.86 \\
\hline MAF10 & 1.28 & 204 & 115.8 & 0.52 & 0.47 \\
\hline MAF11 & 1.77 & 201 & 85.2 & 0.21 & 0.67 \\
\hline MAF11b & 1.77 & 201 & 85.2 & 0.21 & 0.75 \\
\hline MAF12 & 2 & 177 & 81.8 & 0.24 & 0.75 \\
\hline MAF12 & 2 & 177 & 81.8 & 0.24 & 0.75 \\
\hline MAF12b & 2 & 177 & 81.8 & 0.25 & 0.73 \\
\hline MAF13 & 1.4 & 223 & 109.2 & 0.35 & 0.62 \\
\hline MAF14 & 1.73 & 153 & 81 & 0.31 & 0.87 \\
\hline MAF15 & 1.74 & 154 & 82.9 & 0.26 & 0.77 \\
\hline MAF15 & 1.74 & 154 & 82.9 & 0.26 & 0.77 \\
\hline MAF16 & 1.79 & 204 & 99.4 & 0.31 & 0.55 \\
\hline MAF17 & 1.99 & 176 & 109.5 & 0.34 & 0.32 \\
\hline MAF17 & 1.99 & 176 & 109.5 & 0.34 & 0.32 \\
\hline MAF18 & 1.49 & 238 & 108.8 & 0.27 & 0.25 \\
\hline MAF19 & 1.87 & 166 & 110.8 & 0.33 & 0.48 \\
\hline MAF20 & 1.4 & 224 & 98.2 & 0.36 & 0.46 \\
\hline MAF21 & 2.01 & 178 & 97.9 & 0.24 & 0.46 \\
\hline MAF22 & 1.65 & 188 & 84.7 & 0.27 & 0.62 \\
\hline MAF23 & 1.53 & 174 & 97.4 & 0.36 & 0.46 \\
\hline MAF24 & 1.69 & 192 & 106.4 & 0.34 & 0.23 \\
\hline MAF25 & 1.53 & 175 & 110.6 & 0.38 & 0.33 \\
\hline MAF26 & 1.31 & 209 & 97.1 & 0.33 & 0.31 \\
\hline MAF27 & 1.5 & 239 & 85.4 & 0.27 & 0.38 \\
\hline & & & & & \\
\hline
\end{tabular}

